\title{
Morphology of the cesarean section scar in the non-pregnant uterus after one elective cesarean section
}

\author{
Michał Pomorski, Tomasz Fuchs, Anna Rosner-Tenerowicz, Mariusz Zimmer \\ $2^{\text {nd }}$ Department of Gynecology and Obstetrics, Wroclaw Medical University, Poland
}

\begin{abstract}
Objectives: A growing number of studies suggest that the incomplete healing of the CS scar in the uterus increase the risk of uterine dehiscence or rupture during subsequent pregnancies. Thus, the factors that affect wound healing should be evaluated. We aimed to determine whether the morphology of the CS scar in non-pregnant women after one elective CS was affected by the site of the uterine incision, uterine flexion, maternal age, and fetal birth weight.

Material and methods: 208 non-pregnant women were invited for participation in the study, but only 101 of them met inclusion criteria. Standardized scar parameters (residual myometrial thickness (RMT), depth (D) and width (W) of the hypoechoic niche) were measured using ultrasonography at least 6 weeks after the CS.

Results: Scar defect was detected in 26 of 101 subjects. Women without scar defect had significantly higher RMT values (1.87 vs. 0.87), lower newborn birth weight ( $3127 \mathrm{~g}$ vs. $3295 \mathrm{~g}$ ), and higher scar location above the internal cervical os (62\% vs. $16 \%)$, than those with scar defect. Maternal age was significantly correlated with $D$ value $(R=0.40)$. Uterine retroflexion was significantly correlated with a larger $D$ value $(R=0.63)$ and a larger $D / R M T$ ratio $(R=0.24)$.

Conclusions: In low-risk women who have undergone one elective CS, several risk factors are associated with development of the scar defect, but only scar location can be modified during surgery. Future research is needed to determine whether a relatively higher incision location in the uterus can ensure optimal healing of the CS scar.
\end{abstract}

Key words: cesarean section scar, uterine scar, residual myometrial thickness, scar morphology, scar defect, ultrasonography, risk factors of scar defect

Ginekologia Polska 2017; 88, 4: 174-179

\section{INTRODUCTION}

In 2009, more than $26 \%$ of low-risk primiparous women delivered via cesarean section (CS) in the United States [1, 2]. Many initiatives have been undertaken to decrease the rate of primary CS, but the mean CS rate in developed countries is still high (approximately 30\%) [1, 3, 4]. Thus, the population of women who have undergone a primary CS is growing rapidly each year. Many of these women will pursue future pregnancies, and they are at risk of potentially life treating complications of primary CS [5]. These complications include cesarean scar pregnancy, morbidly adherent placenta, and CS scar dehiscence and rupture [2, 5-8]. A growing number of studies suggest that the occurrence of long-term consequences of CS is related to the incomplete healing of the $\mathrm{CS}$ scar in the uterus, i.e., the development of a niche within the scar [7-10]. According to these studies, ultrasonographic assessment of the morphology of the CS scar in women who are not currently pregnant may predict the occurrence of CS scar dehiscence and rupture during subsequent pregnancies. Additionally, cesarean scar pregnancy, a potential precursor of morbidly adherent placenta, mostly develops within the niche of the CS scar $[2,6]$.

Considering the rapidly growing population of women who have undergone a CS and the potential clinical importance of the morphology of the SC scar, the factors that affect wound healing after a CS should be evaluated. Several studies have assessed factors that potentially affect the healing of the CS scar [11-16]. However, to the best of our knowledge, none of these previous studies have assessed CS scar morphology in women who are not pregnant using

Corresponding author:

Michał Pomorski

$2^{\text {nd }}$ Department of Gynecology and Obstetrics, Wroclaw Medical University

Borowska St. 213, 50-556 Wroclaw, Poland

tel.: +48717331400

e-mail: pomorski.md@gmail.com 
standardized ultrasonographic measurements of CS scars in a homogenous group of low-risk women after a primary elective CS. A PubMed search revealed only one study that had assessed the association between the location of the CS scar and its ultrasonographic appearance [13].

The aim of the study was to determine whether the morphology of the CS scar in non-pregnant women after one elective CS was affected by the site of the uterine incision, uterine flexion, maternal age or fetal birth weight. These factors are related to standardized CS scar parameters including the depth of the scar (D) and residual myometrial thickness (RMT) as well as D/RMT ratio.

\section{MATERIAL AND METHODS}

In this case controlled study, which is a part of our prospective study on the prediction of CS scar integrity in subsequent pregnancies, women who delivered by CS at our institution from 2014 to 2016 were invited to undergo ultrasonographic assessment of the CS scar at least 6 weeks after the CS. The study protocol was accepted by the ethics committee on 4 October 2007 (approval no KB 454/2007) and all participants signed the informed consent form before entering the study. The study was conducted in accordance with the 1964 Helsinki declaration and its later amendments.

The examinations were conducted by the first author of this study using a Voluson E8 ultrasound machine (General Electric Medical Systems) with a 4-9 MHz transvaginal convex probe. A standardized approach for imaging and measuring a CS scar via ultrasonography was used, which is described in detail in our previous publications $[7,15]$. For standardization purposes, we used the terms introduced by
Naji et al. [17]. Additionally, an assessment of the location of the CS scar was performed.

The measurements were obtained in the sagittal transection of the uterus. The following standardized scar parameters were assessed: residual myometrial thickness (RMT), depth (D) and the width (W) of the hypoechoic triangle (niche), as shown in Figure 1. The niche was defined according to de Vaate et al. as "any indentation representing myometrial discontinuity at the site of the scar that communicates with the uterine or cervical cavity" [16]. In completely healed CS scars, when the niche was not present, only the RMT value was measured. For the assessment of the location of the CS scar in cases of completely healed CS scars, the distance from the internal cervical os to the lowest demarcation of the RMT was measured. In the presence of a hypoechoic triangle (niche) in the scar, the distance was measured to the proximal edge of the triangle, as it represents the border of the scar (Fig. 1). The inner os of the cervical canal was identified, as in the studies of Vikhareva Osser and Valentin, at the level of the narrowing of the uterus between the corpus and the cervix at the lower margin of the bladder $[13,18]$. Measurements above the inner os of the cervical canal were expressed in positive values, whereas measurements below the inner os, i.e., in the cervical canal, were expressed as negative values.

Clinical information regarding maternal medical history and pregnancy course were collected from medical records and analyzed after ultrasonographic assessment of the CS scar.

The inclusion criteria were as follows: first singleton pregnancy, uneventful pregnancy course, CS performed at term as a planned, elective procedure before the onset of

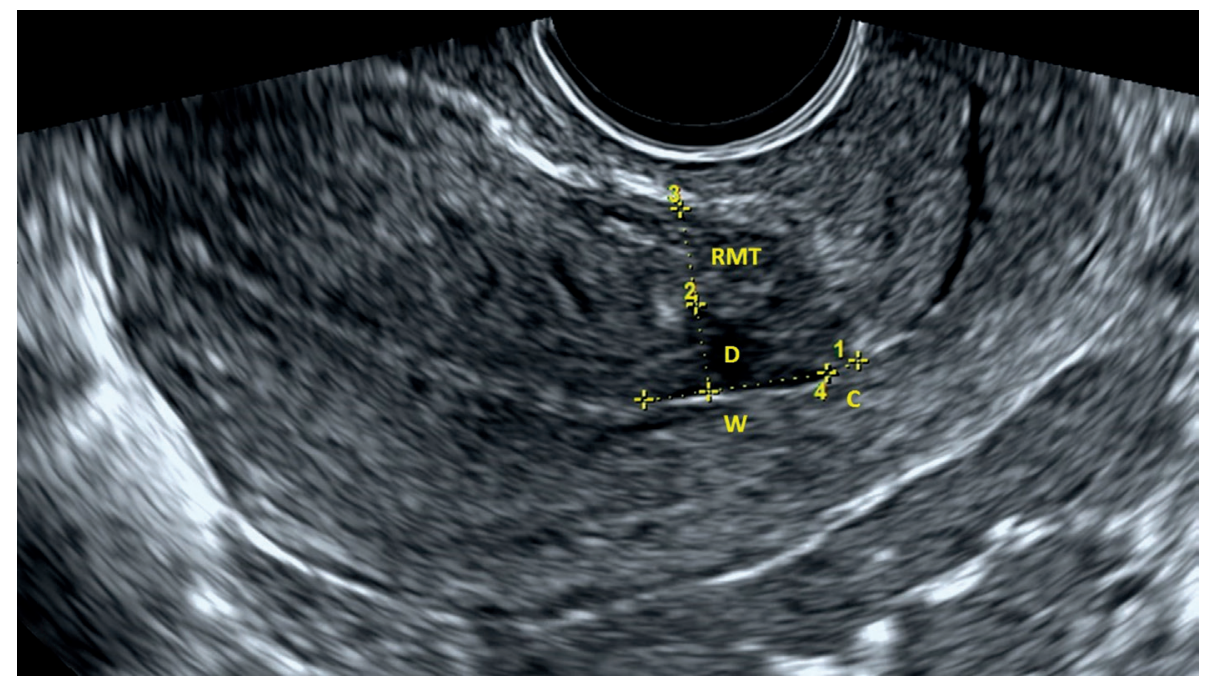

Figure 1. Measurement of the standardized cesarean section scar parameters and assessment of the scar location; RMT — residual myometrial thickness; D - depth of the scar niche; W — width of the scar niche; $\mathrm{C}$ - distance from the internal cervical os to the scar 
uterine contractions, low transverse uterine incision, single layer continuous full thickness uterine closure and uneventful postoperative course. Women with a history of uterine surgery, congenital uterine malformations, diabetes mellitus, hypertension, chronic corticosteroid administration, fetal birth weight under $2500 \mathrm{~g}$ or over $4500 \mathrm{~g}$ and amniotic fluid index (AFI) under 5 or over 20 during the last ultrasound prior to CS were excluded.

\section{Statistical analysis}

The statistical analysis was performed with Statistica software v. 10 (StatSoft, Tulsa, OK, USA). The Mann-Whitney $\mathrm{U}$ test for continuous variables and the chi-square test for categorical data were used to determine the statistical significance of differences between groups. Relationships were analyzed with Spearman's rank correlation coefficient. The criteria for statistical significance was set at $p<0.05$.

\section{RESULTS}

Between the years 2014 and 2016, 208 women participated in this study. From this group, 107 women were excluded, according to the inclusion and exclusion criteria listed above, resulting in 101 patients included in the analysis.

The study group included women with mean age of 32.43 (SD = 3.54) years and gestational age from 37 to 42 weeks (median 38) at CS. Of the 101 participants, 75 presented with an intact cesarean scar, and 26 presented with a scar with a niche. The majority of the studied scars (80\%) were located above the internal cervical os. The mean values of variables such as scar location, RMT and newborn birth weight significantly differed between the groups with and without a scar niche. Detailed characteristics of the study group are presented in Table 1.

Maternal age was significantly positively correlated with scar depth $(R=0.40)$. Newborn birth weight was correlated with the $D / R M T$ ratio $(R=0.20)$ and the presence of the scar niche $(R=0.22)$. The frequency of niche development was decreased in higher locations above the internal cervical os $(R=-0.21 ; p=0.0311)$. Uterine retroflexion was associated with a larger $D$ value and a larger $D / R M T$ ratio $(R=0.63$ for $\mathrm{D} ; \mathrm{R}=0.24$ for $\mathrm{D} / \mathrm{RMT}$ ). Uterine anteflexion was associated with greater distance between the scar and the internal cervical os $(R=-0.26)$. Spearman's rank correlation coefficients between all analyzed variables are presented in Table 2 .

\section{DISCUSSION}

Recently Vervoort et al. summarized the current knowledge of the etiology of incomplete CS scar healing, i.e., the development of the niche [19]. The following characteristics were discussed: a lower location of the CS scar, incomplete closure of the uterine wall, adhesion formation and patient related factors. Potential risk factors for niche development were also presented by de Vaate et al. in a systematic review [16]. Both groups of authors admit that the current evidence regarding factors that affect CS scar healing is limited by

\begin{tabular}{|c|c|c|c|c|}
\hline & Total & Without scar defect & With scar defect & Probability (p) \\
\hline Age, years [mean (SD)] & $32.43(3.54)$ & $32.36(3.75)$ & $32.62(2.89)$ & $0.8926^{*}$ \\
\hline Gestational weeks [mean (SD)] & $38.40(1.10)$ & $38.33(1.04)$ & $38.58(1.24)$ & $0.3802^{*}$ \\
\hline Scar location [n (\%)] & & & & $0.0345^{* *}$ \\
\hline under i.c. os & $4(3.96)$ & $1(0.99)$ & $3(2.97)$ & \\
\hline at i.c. os & $17(16.83)$ & $11(10.89)$ & $6(5.94)$ & \\
\hline above i.c. os & $80(79.21)$ & $63(62.38)$ & $17(16.38)$ & \\
\hline $\begin{array}{l}\text { Distance between the scar and the i.c. os }[\mathrm{cm}] \\
\text { [mean (SD)] }\end{array}$ & $0.56(0.45)$ & $0.58(0.43)$ & $0.50(0.51)$ & $0.2378^{*}$ \\
\hline RMT; [mean (SD)] & $1.03(0.37)$ & $1.08(0.34)$ & $0.87(0.39)$ & $0.0220^{*}$ \\
\hline W, cm; [mean (SD)] & $0.99(0.59)$ & - & $0.99(0.59)$ & - \\
\hline $\mathrm{D}, \mathrm{cm}$; [mean (SD)] & $0.62(0.41)$ & - & $0.62(0.41)$ & - \\
\hline D/RMT; [median, range] & $0(0-6.15)$ & - & $0.58(0.12-6.15)$ & - \\
\hline Uterine position [n (\%)] & & & & $0.0657^{* *}$ \\
\hline Anteflexion & $69(68.32)$ & $55(54.46)$ & $14(13.86)$ & \\
\hline Retroflexion & $32(31.68)$ & $20(19.80)$ & $12(11.88)$ & \\
\hline Newborn birth weight, g; [mean (SD)] & $3171(373)$ & 3127 (359) & 3295 (391) & 0.0293* \\
\hline $\mathrm{AFI} ;[$ mean (SD)] & $7.90(0.90)$ & $7.89(0.91)$ & $7.92(0.89)$ & $0.8804^{*}$ \\
\hline
\end{tabular}

*Mann-Whitney $\mathrm{U}$ test; ${ }^{* *}$ chi-square test; $\mathrm{p}$ values $<0.05$ were regarded as significant and marked in bold. i.c. os — internal cervical os, RMT — residual myometrial thickness, W - width of the scar niche, D — depth of the scar niche, AFI — amniotic fluid index 


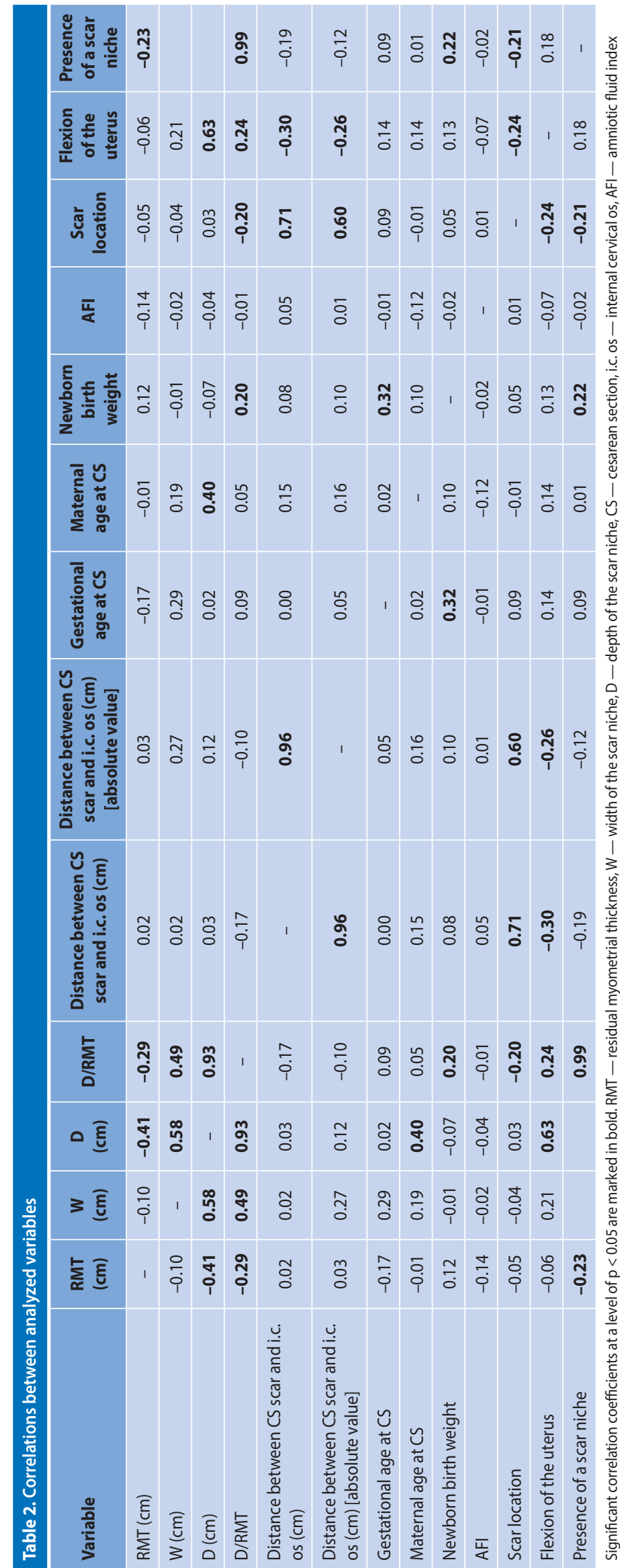

inconsistencies in the methodology of CS scar assessment including different methods of CS scar detection, different definitions of the niche and different patient populations [16, 19].

For two reasons, our study was performed on a homogenous group of low-risk subjects who had received one elective CS. First, this is a rapidly growing population that will most likely pursue future pregnancies and will potentially be at risk of severe obstetrical complications related to the CS scar. Second, the risk factors for deficient CS scar healing in this population and the relationship between the site of the incision and scar morphology have not been studied with a standardized approach for the measurement of CS scars. This method encompasses measurements of both components of the CS scar, namely the RMT and the niche, and thus enables the assessment of the correlation between the studied factors and all scar parameters (RMT, D and W). Additionally, the relationship between the studied factors and the D/RMT ratio was assessed. We included the D/RMT ratio in this analysis because, as we found in our previous study, it was the only parameter of the CS scar measured in the non-pregnant uterus that was useful for predicting CS scar dehiscence during a subsequent pregnancy [7].

Niche prevalence depends on the population of women evaluated and the methodology of scar assessment, and the value varies between $24-70 \%$ [16]. In our study, a niche was found in $25.7 \%$ of women. In a study by Vikhareva Osser et al. of women after one CS, a niche was detected with ultrasonography in $66 \%$ of subjects [18]. In a study by van der Voet, the niche prevalence was $49.6 \%$ [20]. The higher incidence of niche occurrence in these studies may be explained by the fact that Vikhareva Osser et al. included women experiencing active labor and because van der Voet's study was performed on a random population of women after one or multiple CSs. These factors are known to increase the risk of niche development $[16,19]$.

In our study, the correlation between scar morphology and scar location was assessed. The location of the CS scar may be influenced by the development of the lower uterine segment (duration of pregnancy, AFI, fetal weight, stage of the delivery and previous uterine scars). Thus, in this study, only participants 
with a history of one elective CS in a term pregnancy, with a newborn birth weight appropriate for gestational age and a normal amount of amniotic fluid, were included. Additionally, we excluded subjects with other factors that are known or suspected to negatively affect scar healing such as incomplete uterine closure, intrapartum CS, corticosteroid administration, and hypertension [11-14,16].

Only one study was found that assessed the effect of scar location on scar morphology and focused on the measurement of the distance between the CS scar and the inner os of the cervical canal [13].

In the mentioned study, a very low uterine incision was found to be an independent risk factor for the development of large niches. Taking into consideration the definition of large defects used by Vikharvea Osser and Valentin (RMT $\leq 2.2 \mathrm{~mm}$ ), a lower incision was also associated with a decrease in RMT value. In our study, the frequency of niche development decreased with a higher location above the internal cervical os.

Interestingly, in our study of elective CS, 17 participants exhibited a scar localized at the level of the internal cervical os and four participants presented with a CS scar within the cervix. All cervical scars exhibited a niche. In a study by Vikhareva Osser and Valentin that included women in active labor, in $67 \%$ of cases, the top of the scar was located at the level of the internal cervical os, but no cases were reported with the top of the scar located in the cervix [13]. However, in a study by Zimmer et al. that assessed the location of the CS scar in the 14-16 ${ }^{\text {th }}$ week of a subsequent pregnancy, the cervical location of the scar was found in $52 \%$ of women with a history of elective CS and $75 \%$ of women who underwent an intrapartum CS [21]. However, these results cannot be directly compared due to different study designs and methodologies; even in CSs performed without uterine contractions, the incision may extend through cervical tissue. This can be explained by the fact that cervical effacement may begin prior to active labor [21].

In our study of women who had undergone one elective $\mathrm{CS}$, retroflexion of the uterus was found to be associated with a deeper scar niche and a larger D/RMT ratio.

We found three studies that assessed scar morphology in relation to flexion of the uterus. In a study by Vikhareva Osser and Valentin of a group of women who had undergone one CS, large scar niches (defined as RMT $\leq 2.2 \mathrm{~mm}$ ) were more common in subjects with retroflexed uteruses [13]. Ofili-Yebovi et al. found that the likelihood of a woman with a retroflexed uterus developing a niche (defined as myometrial thinning at the site of the scar) was more than $50 \%$ higher than for a woman with an anteflexed uterus [14]. Wang et al. assessed CS scar parameters using a standardized approach. His study included 207 patients, the majority of whom had undergone more than one CS and had a visible niche within the scar. He found that the mean width of the niche was significantly greater in women with a retroflexed uterus than in subjects with an anteflexed uterus [11]. One possible pathophysiological explanation of the incomplete healing of the uterine incision is that the retroflexed uterine corpus causes some degree of tension on the lower uterine segment that negatively affects the approximation and vascular perfusion of the myometrial layers $[14,19]$.

In the current study, maternal age was significantly positively correlated with the depth of the CS scar niche. We found only one study that analyzed maternal age with regard to the size of the scar niche in the uterus of non-pregnant women [13]. In this study, maternal age was found to predict large scar niches. In the study by Naji et al., CS scar dimensions were assessed during pregnancy and older maternal age was associated with a greater increase in the width of the scar niche [22].

To avoid heterogeneity in this study, only subjects with newborns weighing 2500-4500 g were included. Despite this restriction, we found that newborn birth weight was positively correlated with the D/RMT ratio and the presence of a scar niche. This finding indicates that fetal weight in women at term, with a normal amount of amniotic fluid before the onset of uterine contractions, in the absence of multifetal pregnancies, previous uterine surgeries or congenital uterine malformations, may contribute to the development or thinning of the lower uterine segment.

In low-risk women who have undergone one elective CS, the factors that influence CS scar morphology include the distance from the internal cervical os, uterine flexion, fetal birth weight and maternal age. Among these factors, scar location is the only factor related to surgery and can thus be modified.

\section{CONCLUSIONS}

Future research is needed to determine whether a relatively higher incision location in the uterus can ensure optimal healing of the CS scar. Until then, no changes in clinical practice are recommended. However, the establishment of a repeatable cutting plane is difficult, even in cases of elective CS. The cervical-corporal junction in a term pregnancy is difficult to locate, and the development of the lower uterine segment may vary according to the pregnancy duration, size of the uterus, effacement of the cervix and other factors. 


\section{REFERENCES}

1. Spong CY, Berghella V, Wenstrom KD, et al. Preventing the first cesarean delivery: summary of a joint Eunice Kennedy Shriver National Institute of Child Health and Human Development, Society for Maternal-Fetal Medicine, and American College of Obstetricians and Gynecologists Workshop. Obstet Gynecol. 2012; 120(5): 1181-1193, doi: 10.7863/ultra.33.10.1876, indexed in Pubmed: 23090537.

2. Timor-Tritsch IE, Monteagudo A, Cali G, et al. Cesarean scar pregnancy and early placenta accreta share common histology. Ultrasound Obstet Gynecol. 2014; 43(4): 383-395, doi: 10.1002/uog.13282, indexed in Pubmed: 24357257.

3. Caughey AB, Cahill AG, Guise JM, et al. American College of Obstetricians and Gynecologists (College), Society for Maternal-Fetal Medicine. Safe prevention of the primary cesarean delivery. Am J Obstet Gynecol. 2014; 210(3): 179-193, doi: 10.1016/j.ajog.2014.01.026, indexed in Pubmed: 24565430.

4. Boyle A, Reddy UM, Landy HJ, et al. Primary cesarean delivery in the United States. Obstet Gynecol. 2013; 122(1): 33-40, doi: 10.1097/AOG.0b013e3182952242, indexed in Pubmed: 23743454.

5. Clark EAS, Silver RM. Long-term maternal morbidity associated with repeat cesarean delivery. Am J Obstet Gynecol. 2011; 205(6 Suppl): S2-10, doi: 10.1016/j.ajog.2011.09.028, indexed in Pubmed: 22114995.

6. Timor-Tritsch IE, Monteagudo A. Unforeseen consequences of the increasing rate of cesarean deliveries: early placenta accreta and cesarean scar pregnancy. A review. Am J Obstet Gynecol. 2012; 207(1): 14-29, doi: 10.1016/j.ajog.2012.03.007, indexed in Pubmed: 22516620.

7. Pomorski M, Fuchs T, Zimmer M. Prediction of uterine dehiscence using ultrasonographic parameters of cesarean section scar in the nonpregnant uterus: a prospective observational study. BMC Pregnancy Childbirth. 2014; 14: 365, doi: 10.1186/s12884-014-0365-3, indexed in Pubmed: 25733122.

8. Valentin L. Prediction of scar integrity and vaginal birth after caesarean delivery. Best Pract Res Clin Obstet Gynaecol. 2013; 27(2): 285-295, doi: 10.1016/j.bpobgyn.2012.09.003, indexed in Pubmed: 23103207.

9. Vikhareva Osser O, Valentin L. Clinical importance of appearance of cesarean hysterotomy scar at transvaginal ultrasonography in nonpregnant women. Obstet Gynecol. 2011; 117(3): 525-532, doi: 10.1097/AOG.0b013e318209abfo, indexed in Pubmed: 21343754.

10. Varner M. Cesarean scar imaging and prediction of subsequent obstetric complications. Clin Obstet Gynecol. 2012; 55(4): 988-996, doi: 10.1097/GRF.0b013e318263c699, indexed in Pubmed: 23090468.

11. Wang CB, Chiu WW, Lee CY, et al. Cesarean scar defect: correlation between Cesarean section number, defect size, clinical symptoms and uterine position. Ultrasound Obstet Gynecol. 2009; 34(1): 85-89, doi: 10.1002/uog.6405, indexed in Pubmed: 19565535.

12. Yazicioglu F, Gökdogan A, Kelekci S, et al. Incomplete healing of the uterine incision after caesarean section: Is it preventable? Eur J Obstet Gynecol Reprod Biol. 2006; 124(1): 32-36, doi: 10.1016/j.ejogrb.2005.03.023, indexed in Pubmed: 16023780.

13. Vikhareva Osser O, Valentin L. Risk factors for incomplete healing of the uterine incision after caesarean section. BJOG. 2010; 117(9): 1119-1126, doi: 10.1111/j.1471-0528.2010.02631.x, indexed in Pubmed: 20604776.

14. Ofili-Yebovi D, Ben-Nagi J, Sawyer E, et al. Deficient lower-segment Cesarean section scars: prevalence and risk factors. Ultrasound Obstet Gynecol. 2008; 31(1): 72-77, doi: 10.1002/uog.5200, indexed in Pubmed: 18061960.

15. Zimmer M, Pomorski M, Fuchs T, et al. Ultrasonographic analysis of cesarean scars features in nonpregnant uterus. Ginekol Pol. 2007; 78(11): 842-846, indexed in Pubmed: 18306913.

16. Bij de Vaate AJM, van der Voet LF, Naji O, et al. Prevalence, potential risk factors for development and symptoms related to the presence of uterine niches following Cesarean section: systematic review. Ultrasound Obstet Gynecol. 2014; 43(4): 372-382, doi: 10.1002/uog.13199, indexed in Pubmed: 23996650.

17. Naji O, Abdallah Y, Bij De Vaate AJ, et al. Standardized approach for imaging and measuring Cesarean section scars using ultrasonography. UItrasound Obstet Gynecol. 2012; 39(3): 252-259, doi: 10.1002/uog.10077, indexed in Pubmed: 21858885.

18. Osser OV, Jokubkiene L, Valentin L. High prevalence of defects in Cesarean section scars at transvaginal ultrasound examination. Ultrasound Obstet Gynecol. 2009; 34(1): 90-97, doi: 10.1002/uog.6395, indexed in Pubmed: 19499514.

19. Vervoort A, Uittenbogaard LB, Hehenkamp W, et al. Why do niches develop in Caesarean uterine scars? Hypotheses on the aetiology of niche development. Hum Reprod. 2015: dev240, doi: 10.1093/humrep/dev240.

20. Zimmer EZ, Bardin R, Tamir A, et al. Sonographic imaging of cervical scars after Cesarean section. Ultrasound Obstet Gynecol. 2004; 23(6): 594-598, doi: 10.1002/uog.1033, indexed in Pubmed: 15170802.

21. van der Voet LF, Bij de Vaate AM, Veersema S, et al. Long-term complications of caesarean section. The niche in the scar: a prospective cohort study on niche prevalence and its relation to abnormal uterine bleeding BJOG. 2014; 121(2): 236-244, doi: 10.1111/1471-0528.12542, indexed in Pubmed: 24373597.

22. Naji O, Daemen A, Smith A, et al. Changes in Cesarean section scar dimensions during pregnancy: a prospective longitudinal study. Ultrasound Obstet Gynecol. 2013; 41(5): 556-562, doi: 10.1002/uog.12334, indexed in Pubmed: 23108803. 